\title{
EVALUACIÓN DE LA INFLUENCIA DE LA REVALUACIÓN DEL TIPO DE CAMBIO SOBRE LA DEMANDA DE TURISMO EN COLOMBIA
}

\author{
RAÚL QUEJADA PÉREZ* \\ FELIPE DEL RÍO CARRASQUILLA*
}

\section{RESUMEN:}

El turismo representa una actividad fundamental dentro de una economía, por los diferentes impactos que tiene sobre otros sectores. En el mundo se está viviendo cierto auge en la actividad turística, reflejando Colombia similares tendencias en esta materia. Recientemente se está presentando un fenómeno revaluativo del tipo de cambio peso-dólar, aspecto este que motiva al estudio de tal situación, e indagar la influencia que tiene esta variable sobre la demanda de exportaciones y de importaciones turísticas, lo que constituye el objeto de estudio del presente trabajo. El método de MCO (Minimos Cuadrados Ordinarios) es la herramienta que permite la consecución de tal propósito, luego de verificar la existencia de raíces unitarias. Finalmente los resultados obtenidos permiten concluir que para los turistas extranjeros los precios relativos no resultan ser determinantes al decidir considerar a Colombia como destino turístico y que los colombianos que deciden efectuar turismo en el exterior están aprovechando el contexto revaluativo favorable, que se refleja en las progresivas disminuciones en el nivel de precios internos.

Palabras Clave: turismo, demanda, revaluación, precios relativos

Clasificación JEL: L83, C32

\section{ABSTRACT:}

The tourism represents a fundamental activity inside an economy, for the different impacts that it has on other sectors. In the world one is living certain peak in the tourist activity, reflecting Colombia similar tendencies in this matter. Recently it is showing up a phenomenon revaluativo of the exchange rate peso-dollar, aspect this that motivates to the study of such a situation, and the influence that has this va-

\footnotetext{
* Magíster en Economía, Decano de la Facultad de Ciencias Económicas de la Universidad de Cartagena, Cartagena (Colombia). Líder del Grupo de Investigación: MERCADO LABORAL UNIVERSIDAD DE CARTAGENA. Correo Electrónico: fceconomicas@unicartagena.edu.co.

** Estudiante de VII Semestre del Programa de Economía de la Universidad de Cartagena. Perteneciente al semillero de investigación adscrito al Grupo MERCADO LABORAL UNIVERSIDAD DE CARTAGENA. Correo electrónico: fdelrioc@gmail.com.
} 
riable on the demand of exports to investigate and of tourist imports, what constitutes the object of study of the present work. The method of MCO (Square Ordinary Minima) is the tool that allows the attainment of such a purpose, after verifying the existence of unitary roots. Finally the obtained results allow to conclude that for the foreign tourists the relative prices don't turn out to be decisive when deciding to consider to Colombia like tourist destination and that the Colombians that decide to make tourism in the exterior taking advantage the context favorable revaluativo that is reflected in the progressive decreases in the level of internal prices.

Key Words: tourism, demand, revaluation, relative prices.

JEL Classification: L83, C32 


\section{INTRODUCCIÓN}

La actividad turística brinda diversas oportunidades para el crecimiento y el desarrollo económico-social de la zona geográfica en que se desarrolle, por esto resulta de vital importancia que tales oportunidades se aprovechen eficientemente en beneficio del sector de interés. Para ello se debe conocer el contexto, sus características y tipicidades en que la actividad tiene lugar, junto con sus naturales interrelaciones en términos de los elementos que dicho análisis involucra, por tanto resulta preciso caracterizarla con el fin de detectar elementos que coadyuven al crecimiento del sector.

La elaboración de este documento tiene como propósito fundamental, construir una herramienta que facilite la toma de decisiones, la planeación y la emisión de juicios por parte de las personas directamente involucradas en el sector, se aborda en este trabajo una temática de gran interés: la revaluación del tipo de cambio; más precisamente se busca evaluar la influencia que ésta ha mostrado sobre la demanda del "producto" turismo.

Lo anterior se hace en doble vía: por un lado se evalúa la influencia que ha tenido la revaluación del tipo de cambio dólar-peso sobre la demanda de turismo nacional por parte de los extranjeros, por otro, se estudia lo anterior pero sobre la demanda de los residentes nacionales colombianos por el producto turístico extranjero.

Para el cumplimiento del objetivo del presente artículo se recurre al sencillo, aunque práctico, método de los Mínimos Cuadrados Ordinarios que se opera sobre dos modelos doblelogarítmicos en diferencias de demanda turística, cuyos parámetros se estiman de manera independiente.

El texto se organiza en varias partes: la primera de ellas hace alusión al marco referencial teórico sobre el tema, la segunda muestra la dinámica reciente de los hechos turísticos en los ámbitos nacional e internacional y la ultima parte se refiere al modelo econométrico planteado para efectos de conocer los elementos determinantes de demanda por exportaciones turísticas colombianas con sus resultados, conclusiones y anexos correspondientes.

\section{MARCO REFERENCIAL}

\section{ANTECEDENTES}

En los trabajos de Mantero, Perelmuter y Sueiro (2004) se estudia la demanda de turismo receptivo en Uruguay, para ello consideran la estima- 
ción de dos modelos ${ }^{1}$ de frecuencias mensuales, uno de carácter agregado y otro que se considera de forma desagregada en cuenta de forma desagregada la procedencia de los turistas, con el propósito de recoger heterogeneidades y "[...] características específicas de los turistas [...] [que pueden quedar] diluidas en un único indicador" (Mantero, Perelmuter y Sueiro 2004, 7). Los autores consideran como variables explicativas del número de turistas extranjeros que recibe Uruguay, el nivel de ingreso del país de procedencia, los precios relativos Uruguay-país de procedencia y los precios relativos del país de procedencia frente a otros destinos alternativos.

\section{MARCO TEÓRICO}

La forma funcional para la especificación de modelos de demanda turística generalmente es de tipo neoclásico (función Cobb-Douglas linealizada), tal como lo sugieren y desarrollan Rodríguez et al. (2003), Aguilera y Galvis (1999), Halicioglu, F. (2004) y Espasa, Gómez y Morales (1993). El primero de los trabajos precitados modela sobre la variable dependiente ingreso generado por las actividades turísticas, dado que los autores consideran que es a través de tal variable con que se miden los resultados de la actividad turismo, antes que con la cantidad de viajeros reportadas. Aguilera y Galvis (1999) para el caso colombiano, y más precisamente para el cartagenero deciden incluir como variable explicada a la cantidad de viajeros entrados a la ciudad, dado que la información, tal que discriminara turistas netos no estaba disponible. El total de turistas arribados a Turquía es la variable dependiente de Halicioglu, F. (2004)². Finalmente Espasa, Gómez y Morales (1993) deciden efectuar un análisis tanto vía ingresos turísticos como vía cantidades de turistas.

La literatura existente alusiva al tema turístico, sobre todo Espasa, Gómez y Morales (1993), recomienda trabajar con modelos de demanda desagregados pues sus "componentes incorpora[n] conjuntos diversos de bienes cuyas funciones de demanda pueden tener elasticidades de renta y precios suficientemente diferentes, de modo que con su agregación se obtiene un conjunto de comportamiento [...] heterogéneo" (p 637). La evidencia, de acuerdo con Mantero, Perelmuter y Sueiro (2004), lo confirma para Uruguay.

${ }^{1}$ A través de técnicas de cointegración y VAR con mecanismos de corrección de errores.

2 Resultan interesantes las breves consideraciones que el autor hace sobre la naturaleza teórica y los resultados empíricos, que según otros autores hay que tener presentes a la hora de estudios que aborden al turismo. 
Otra recomendación dada por los autores mencionados arriba, consiste en que se deben incluir dos índices de competitividad, en el caso en que una nación tenga como competencia países diferentes a aquellos que demandan sus productos y servicios turísticos Espasa, Gómez y Morales (1993, 637); en el presente estudio, dadas las características del mercado turístico colombiano: la competencia es a la vez demandante importante de turismo, se incluirá entonces un solo indicador de competitividad.

Finalmente, para efectos de considerar otras alternativas que permitan realizar futuras indagaciones sobre la influencia del tipo de cambio en la demanda de exportaciones e importaciones turísticas, se sugiere acudir al efecto transmisión del tipo de cambio como referente teórico importante que podría explicar el propósito de interés.

\section{DINÁMICA RECIENTE DE LOS HECHOS DEL TURISMO}

\section{ÁMBITO INTERNACIONAL}

La Organización Mundial de Turismo reportó que para la coyuntura turística internacional del año 2005, se superaron los pronósticos que se tenían. Mundialmente, las llegadas de turistas internacionales rondaron los 800 millones, 42 millones por encima del año inmediatamente anterior, es decir hubo un crecimiento de alrededor 10\% (OMT 2006, 2-3).

Alemania, EE.UU. y el Reino Unido, en su orden, encabezaron el ranking de los que más gasto destinaron en actividades relacionadas con el turismo. De los US $\$ 633.000$ totales, el 11,2\% corresponde al primer país, el 10,4\% al segundo y el $8,9 \%$ al tercero.

En la gráfica 1 se muestra el incremento presentado en el gasto en turismo a nivel mundial. Nótese que para el período 2004-2003 se evidencia un promedio de crecimiento mayor (19,6\%) que para el período 2002-2003 (12,5\%), siendo Australia $(42,2 \%)$, India $(41,6 \%)$ y España $(34,2 \%)$, los países con mayores crecimientos. 
GRÁFICA No. 1. INCREMENTO EN EL GASTO TURÍSTICO INTERNACIONAL. 20032002, 2004-2003.

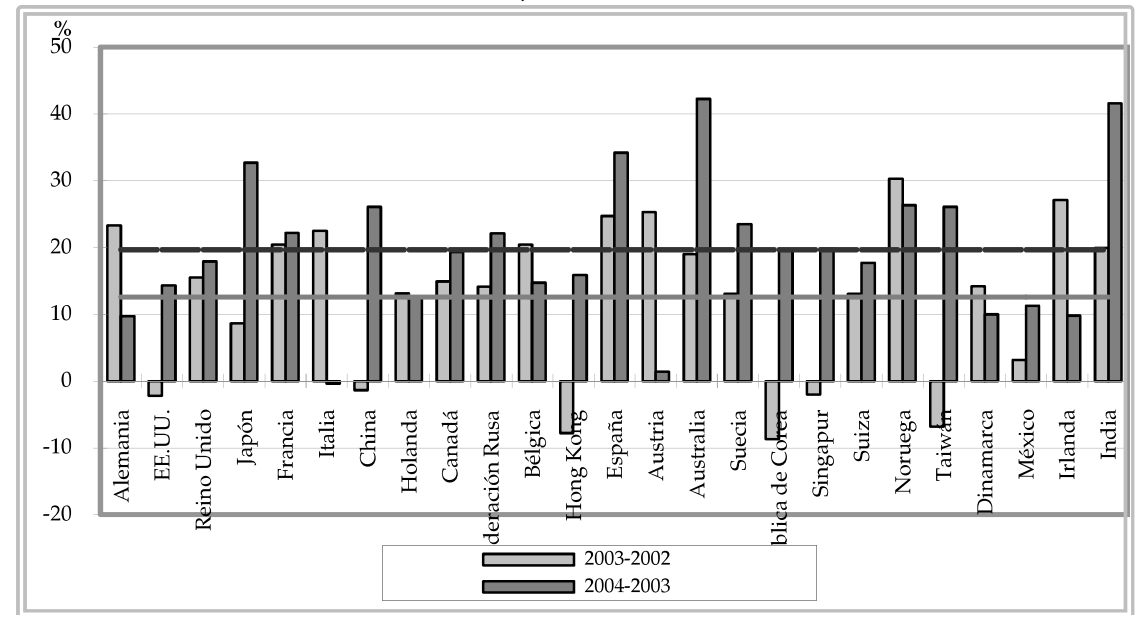

Fuente: WTO, cálculos y diseño de los autores.

El continente americano mostró en 2006 un crecimiento en la actividad turística, que rondó el orden del 6\%. Los países que estuvieron por encima del promedio fueron EE.UU. (7\%), México (6\%) y Cuba (12\%). América del Sur fue una zona en "donde la mayoría de los países se beneficiaron de unos tipos de cambio favorables, una gama más amplia de productos turísticos de calidad, una mayor capacidad aérea y la recuperación económica de mercados emisores cruciales" (OMT 2006, 8), esto condujo a que el crecimiento en 2005 hubiera estado en cerca del 12\%.

\section{ÁMBITO NACIONAL}

En aras de efectuar una caracterización de la actividad turística del lado de la demanda, el Departamento Administrativo Nacional de Estadística de Colombia (DANE), efectuó en el año 2003 la Encuesta de turismo a hogares, que provee cantidad de datos referidos a la actividad turística que realizaron, durante el período de análisis ${ }^{3}$, las personas y los hogares colombianos en términos de gastos y viajes.

A continuación se presentan los 15 principales países de origen de los extranjeros que visitan a Colombia (Tabla 1), así como los 15 países conside-

\footnotetext{
3 Respecto al marco temporal, la ficha técnica de la encuesta indica que: "para viajes [el período de referencia es de]: un año (agosto 2002 a julio 2003); para viajes a segunda vivienda y excursionismo: mes anterior al de recolección (julio 2003); para actividades y grado de satisfacción de servicios: último viaje (indistintamente de la fecha de realización)". DANE $(2007,14)$.
} 
rados como destinos preferidos por los colombianos (Tabla 2)4. Nótese que los 5 primeros países que demandan mayor cantidad de turismo nacional están dentro del grupo de los países más visitados por los colombianos.

GRÁFICA No. 2. PARTICIPACIÓN SEGÚN GRUPOS DE PAÍSES DE PROCEDENCIA DE VIAJEROS EXTRANJEROS QUE VISITAN COLOMBIA. 15 PRINCIPALES PAÍSES AGRUPADOS.

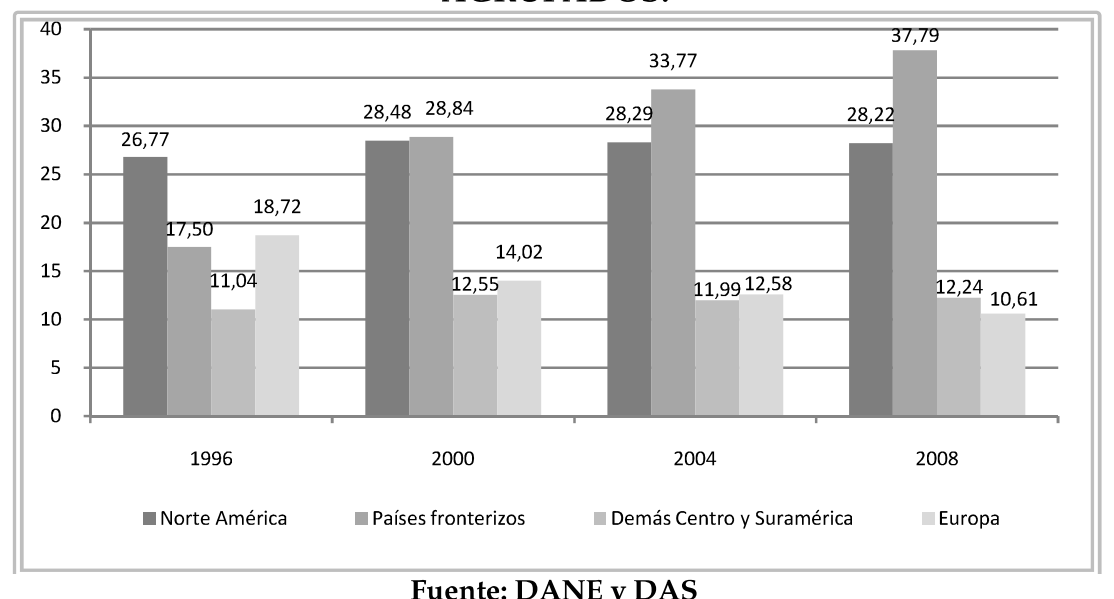

No obstante, del peso significativo que los Estados Unidos tiene como demandante del turismo colombiano se destacan los países fronterizos (Venezuela, Ecuador, Perú, Bolivia) que en su conjunto superan a la demanda de los Estados Unidos, aspecto este que sugiere una especial atención en la elaboración de las políticas económicas con relación al turismo extranjero.

Las temporadas turísticas son un hecho conocido, e importante es tenerlo en cuenta a la hora de efectuar análisis que involucren el estudio de la dinámica del sector. En la gráfica 3 se muestra una relación entre el porcentaje de viajes realizados por turismo en las principales ciudades colombianas y los meses a los que corresponden tales porcentajes. Se denota un comportamiento estacional de la actividad turística donde se observan los picos expansivos más altos de la distribución (temporadas turísticas) se presentan en los meses de Diciembre y Julio, que son coincidentes con el período de vacaciones escolares e institucionales; y en Abril hallarían explicación por la época de Semana Santa. Los picos depresivos de la distribu-

${ }^{4}$ Específicamente las cantidades mostradas en las Tablas 1 y 2, hacen referencia al número de pasajeros llegados por vía aérea, no sólo por motivos de turismo. Así, esta variable se considera proxy de la demanda (tanto externa como interna) de turismo. Participación según grupos de países de procedencia de viajeros extranjeros que visitan Colombia. 15 principales países agrupados. 
ción se observan al final del periodo ocasional del año, Febrero, Mayo y Octubre.

\section{GRÁFICA No. 3. COLOMBIA. PORCENTAJES DE VIAJES TURÍSTICOS POR CIUDAD, SEGÚN MES DE REALIZACIÓN. AGOSTO DE 2002 - JULIO DE 2003.}

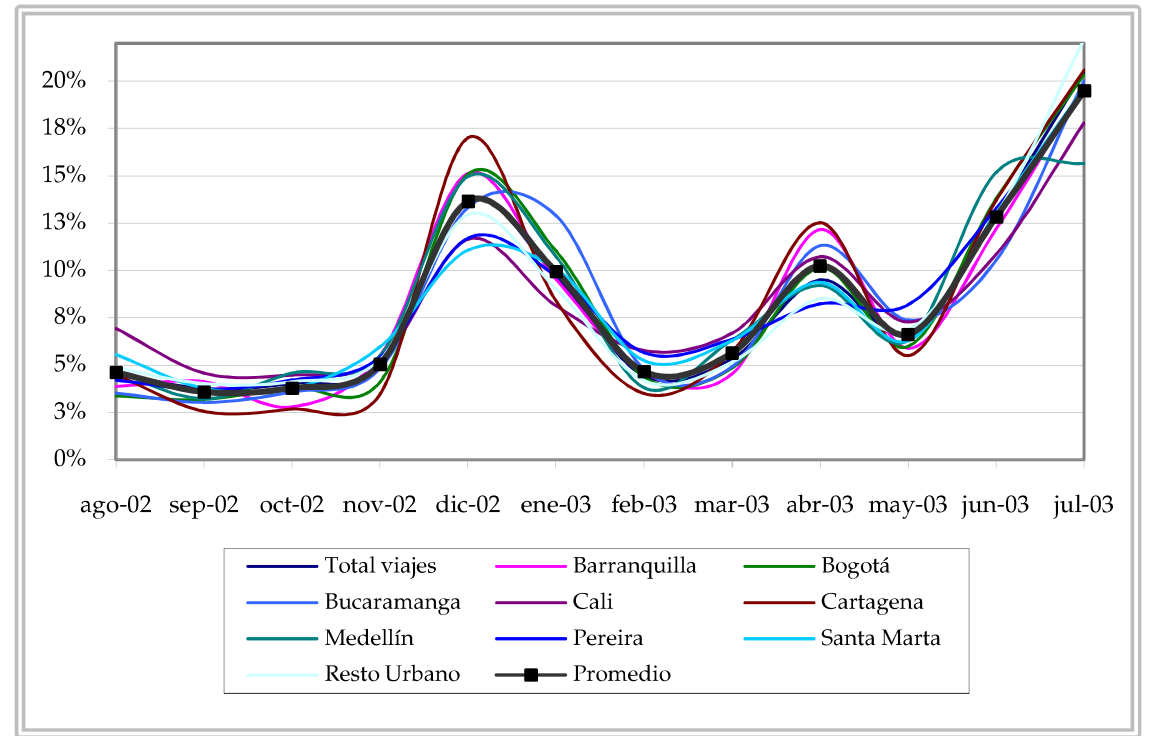

Fuente: DANE. Análisis de los resultados de la Encuesta de Turismo a Hogares 2003. Diseño y cálculos del equipo de trabajo.

Otra forma de cuantificar la demanda de turismo, corresponde a considerar el monto monetario de las exportaciones (realizadas por extranjeros) y las importaciones (realizadas por colombianos) ${ }^{5}$ que están registradas en las cuentas de transporte y viajes que aparecen en la balanza de pagos ${ }^{6}$. En la gráfica 4 las series evidencian una tendencia creciente en el tiempo: las ex-

${ }^{5}$ Aunque es proxy de la demanda, se empleará como variable explicativa en los modelos de demanda de exportaciones e importaciones de turismo que se estimarán más adelante en este documento.

${ }^{6}$ Según la quinta edición del Manual de Balanza de Pagos del FMI, transportes "abarcan la mayoría de los servicios prestados por residentes a no residentes, y viceversa, transporte de carga y de pasajeros por todos los medios, así como otros servicios de distribución y auxiliares, incluido el arrendamiento de equipo de transporte tripulado, con ciertas excepciones [...]" y viajes "comprenden bienes y servicios [...] adquiridos en una economía por viajeros no residentes (incluidos los excursionistas) para fines de negocios y para uso personal durante su estancia (inferior a un año) en esa economía. En viajes no se incluyen los servicios de transporte internacional de pasajeros, que forman parte de transportes. Los estudiantes y las personas que están bajo tratamiento médico se tratan como viajeros, independientemente de la duración de su estancia, en tanto que otros individuos — personal militar y de embajadas y trabajadores no residentes - no se consideran viajeros". CEPAL (2007, 377-378). 
portaciones pasaron de US $\$ 353,63$ millones en el primer trimestre de 1999 a US $\$ 641,4$ millones en el segundo trimestre de 2007; es decir, la tasa promedio de crecimiento de las exportaciones fue de $2,23 \%$ desde el primer al último período de observación. Las importaciones de turismo exhibieron una dinámica similar: en el primer trimestre de 1999 se reportaron US\$ 507,14 millones, mientras que en el segundo de 2007 se tuvieron US\$957,12 millones, siendo la tasa promedio de crecimiento de $2,38 \%$.

GRÁFICA No. 4. COLOMBIA. EXPORTACIONES E IMPORTACIONES DE TURISMO. I TRIMESTRE DE 1999 - II TRIMESTRE DE 2007.

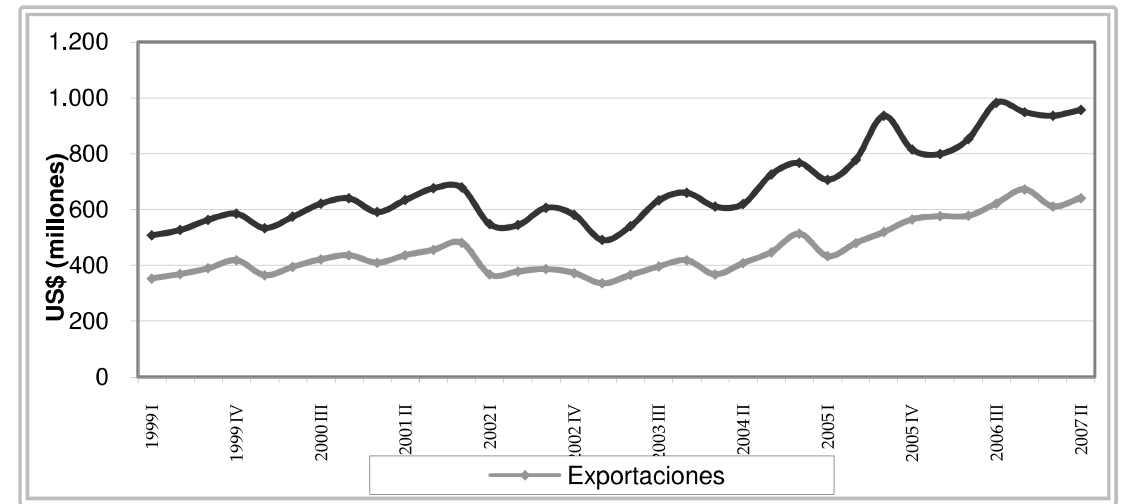

Fuente: Banco de la República. Diseño de los autores.

El comportamiento de la demanda de turismo tanto externa como interna, podría ser producto de la situación económica mundial, en el sentido de que los resultados de crecimiento económico positivo reportados permiten y estimulan un mayor gasto en actividades como las turísticas. La gráfica 5 relaciona en el tiempo la tasa de crecimiento promedio del producto real de los principales 15 países demandantes de turismo y de Colombia. Ésta muestra generalmente signo positivo en los trimestres más recientes, así que podría pensarse en una relación de dependencia entre ambas variables. 
GRÁFICO No. 5. COLOMBIA Y PRINCIPALES 15 PAÍSES DEMANDANTES DE TURISMO. TASA DE CRECIMIENTO DEL PIB REAL. I TRIMESTRE DE 1999 - II TRIMESTRE DE 2007.

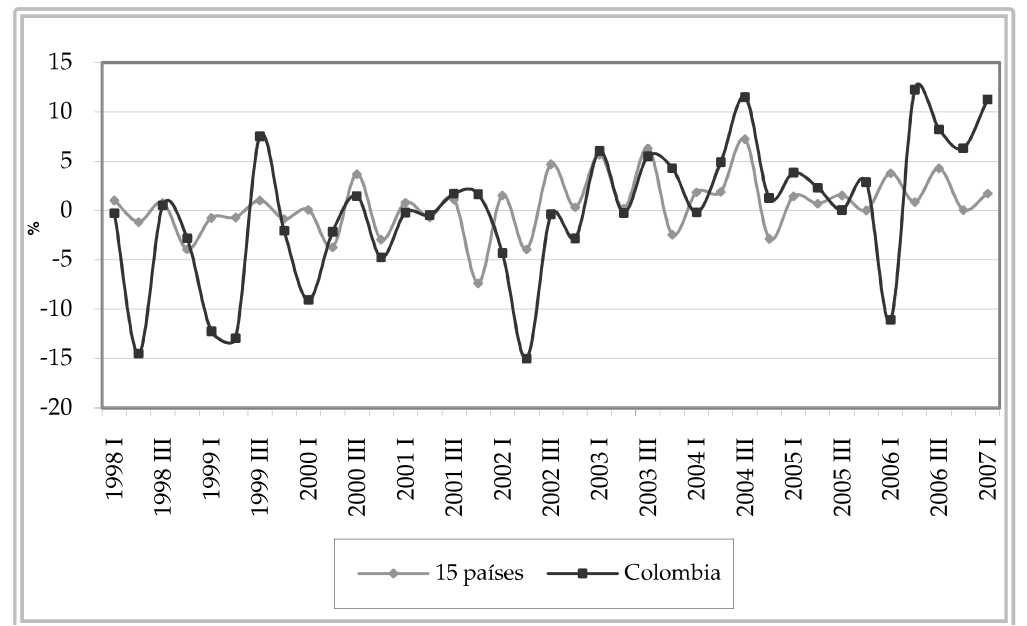

Fuente: International Financial Statistics (IFS) del FMI. Cálculos y diseño de los autores.

\section{Tasa de cambio}

Es sabido que, en la medida en que el tipo o tasa de cambio se revalúa (o aprecia, en un sistema de tipo de cambio flexible como el de Colombia), se entregarán menos unidades monetarias nacionales por cada unidad de moneda extranjera que se traiga al país, lo inverso sucede si el tipo de cambio se devalúa o deprecia.

Según el Banco de la República $(2007,54)$ el peso (con respecto al dólar estadounidense) ha estado presentando un proceso revaluativo (apreciativo) desde mediados de 2003, seguido de un breve período de devaluación durante el segundo trimestre de 2006; más recientemente la tasa de cambio ha caído desde el segundo semestre de 2006 hasta lo que va corrido de 2007. $\mathrm{Al}$ comienzo del primer período apreciativo (octubre 2003) el dólar en Colombia se cotizaba en $\$ 2.873,95$ para finalizar en $\$ 2.261,92$ (marzo 2006). Durante un corto trimestre de recuperación se llegó a cotizar en $\$ 2.542,22$ (junio 2006) y de ahí hasta junio de 2007 tocó fondo la cotización: \$1.923,53 por dólar. Lo mencionado se ilustra trimestralmente en la Gráfica 6. 
GRÁFICA No. 6. COLOMBIA. TASA DE CAMBIO PESO-DÓLAR. I TRIMESTRE DE 1999- II TRIMESTRE DE 2007.

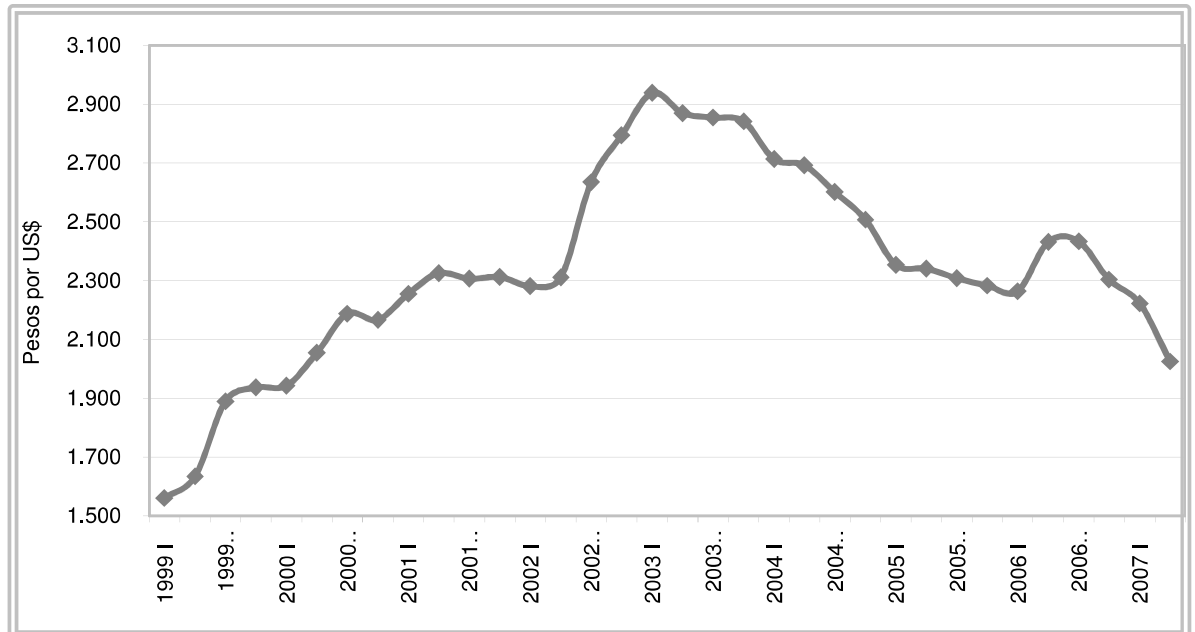

Fuente: Banco de la República, diseño de los autores.

\section{Índice de precios relativos}

Sería interesante y en gran medida útil contar con un indicador de precios relativos de la actividad turística, que recogiera, expresara y permitiera comparar el nivel de precios de Colombia con su principal competencia en el sector, que corresponde a EEUU, México, Canadá, Brasil y Argentina7. En tal sentido se procedió a construir un índice de tasa de cambio real (competitividad) de la actividad turística para Colombia y los países anteriores, siguiendo la metodología del Banco de la República para Colombia (Huertas et al., 2003). En el Anexo 1 se presenta el proceso operativo de construcción del ITCR. El gráfico 7 presenta el índice construido para el período objeto de estudio.

7 El criterio consintió en elegir aquellos países Americanos cercanos a Colombia que reportaran las mayores llegadas de turistas internacionales, que corresponden a 37\%, 16,4\%, $13,9 \%, 4 \%$ y $2,9 \%$ respectivamente, tal como aparece en OMT $(2006,8)$. 
GRÁFICO No. 7. ÍNDICE DE TASA DE CAMBIO REAL ENTRE COLOMBIA Y LOS 5 PAÍSES COMPETENCIA. I TRIMESTRE DE 1999 - II TRIMESTRE DE 2007. $(2000=100)$.

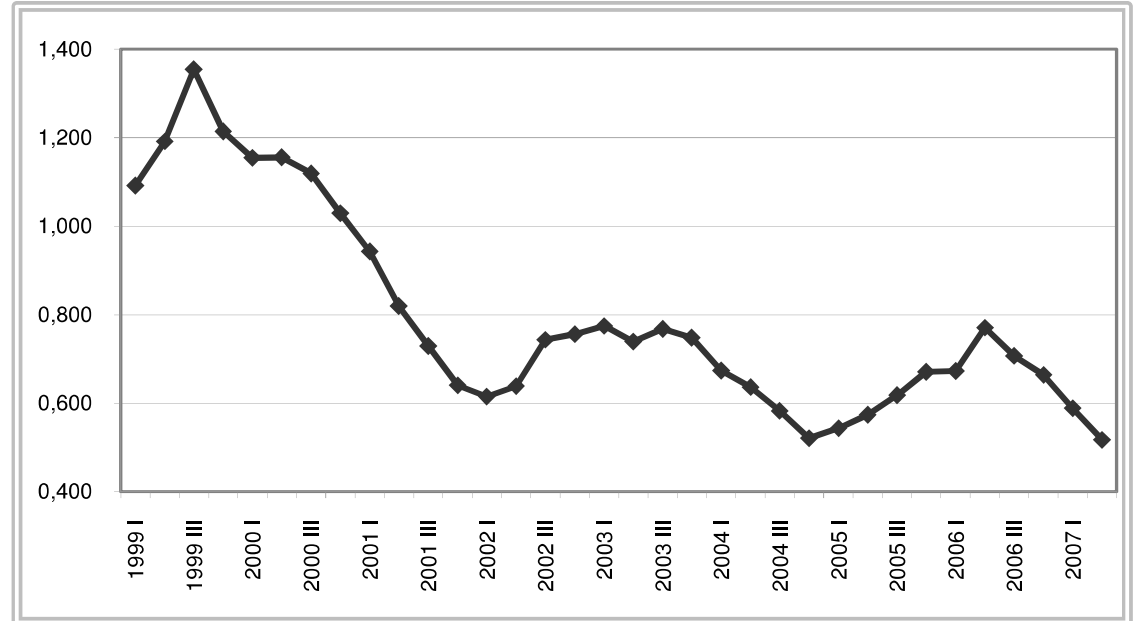

Fuente: Banco de la República, IFS del FMI, DANE (Año de la Encuesta) y DAS. Cálculo y diseño de los autores.

\section{METODOLOGÍA}

En esta sección se presenta formalmente la herramienta con la que se efectuará el análisis del impacto de la revaluación del Peso sobre la demanda de turismo, tanto extranjera en Colombia, como colombiana en el exterior. En el primer apartado se considera el modelo econométrico, en el siguiente se hace la verificación de estacionariedad en las variables para proceder luego a la estimación de los parámetros mediante MCO.

\section{MODELO ECONOMÉTRICO}

La modelación que se efectúa en este trabajo es similar a las llevadas a cabo por Galvis y Aguilera (1999) y Espasa, Gómez y Morales (1993), en tanto que se plantean dos ecuaciones que serán estimadas de forma independiente: una ecuación de demanda de exportaciones del turismo colombiano por parte de los extranjeros, y otra de importaciones de turismo extranjero por parte de colombianos. Respectivamente las ecuaciones (1) y (2) muestran lo anterior.

$$
\begin{gathered}
\ln \mathrm{Qx}_{\mathrm{t}}=\alpha_{0}+\alpha_{1} \ln \mathrm{ITCR}_{\mathrm{t}}+\alpha_{2} \ln \mathrm{ITCR}_{\mathrm{t}-1}+\alpha_{3} \ln \mathrm{Yx}_{\mathrm{t}} \\
+\alpha_{4} \ln \mathrm{Yx}_{\mathrm{t}-1}+\mathrm{D}_{1} \mathrm{~T}_{1}+\mathrm{D}_{2} \mathrm{~T}_{3}+\mathrm{D}_{3} \mathrm{~T}_{4}+\varepsilon_{\mathrm{t}} \\
\ln \mathrm{Qimp}_{\mathrm{t}}=\beta_{0}+\beta_{1} \ln \mathrm{ITCR}_{\mathrm{t}}+\beta_{2} \ln \mathrm{ITCR}_{\mathrm{t}-1}+\beta_{3} \ln \mathrm{Yn}_{\mathrm{t}} \\
+\beta_{4} \ln \mathrm{Yn}_{\mathrm{t}-1}+\mathrm{D}_{1} \mathrm{~T}_{1}+\mathrm{D}_{2} \mathrm{~T}_{3}+\mathrm{D}_{3} \mathrm{~T}_{4}+\varepsilon_{\mathrm{t}}
\end{gathered}
$$

Siendo $\ln$ Qx y $\ln$ Qimp las variaciones de las cantidades exportadas e im- 
portadas de turismo, que se calcularon de los montos de las cuentas de transporte y viajes de la balanza de pagos. Para contar con una medida de los precios relativos entre Colombia y sus principales países competencia en turismo, se construyó un índice de tipo de cambio real ( $\ln$ ITCR ). En este punto no fue necesaria la inclusión de dos índices de competitividad, cosa que se hace "ante situaciones en las que se vende a un conjunto de países pero se compite con otro conjunto distinto de países, al menos en parte sustancial [...]"8 (Espasa, Gómez y Morales 1993, 637). Como se ha evidenciado a lo largo de este documento, los principales países competencia turística para Colombia son a la vez demandantes del "producto"

Se construyeron dos variables índice del nivel de renta real siguiendo a Espasa, Gómez y Morales (1993, 650-651, 654), una que recogía a los principales países demandantes de turismo colombiano $(\ln Y x)$, y otra que representaba el nivel de renta de Colombia $(\ln Y n)$. El proceso de construcción se detalla en el anexo 1 . El término $\varepsilon_{t}$ corresponde a una perturbación estocástica. Finalmente, como lo que se tiene es un modelo doblelogarítmico, los coeficientes $\alpha_{i}$ y $\beta_{i}$ de cada ecuación permiten conocer las elasticidades promedio de las cantidades demandadas de turismo respecto a cada una de las variables.

Cada una de las variables anteriores están expresadas tanto en el momento $t$ como en el $t-1$, dado que los individuos basan sus decisiones presentes, en este caso las asociadas al turismo, en información pasada. En este documento se opera con una frecuencia trimestral, y como se incluye un rezago temporal ${ }^{10}$ se tiene que las series entonces comprenden desde el II trimestre de 1999 al I trimestre de 2007.

Como lo sugiere la gráfica 3, se debe incluir en las ecuaciones un componente que recoja los efectos estacionales; con este fin se adicionan las variable dicótomas $T_{1}, T_{3}$ y $T_{4}$, representaciones de los trimestres primero, tercero y cuarto. Los signos esperados y las fuentes de las variables se muestran en el cuadro 1.

\footnotetext{
${ }^{8}$ En el texto original parte de esta cita está en cursiva.

9 Obsérvese la tabla 1 y el apartado Índice de precios relativos.

10 Más adelante se mostrará que esta es la mejor especificación, pues al incluir más rezagos de las variables el modelo no mejora.
} 
CUADRO No. 1. VARIABLES, SIGNOS ESPERADOS Y FUENTES DE INFORMACIÓN.

\begin{tabular}{|c|c|c|c|c|}
\hline \multicolumn{2}{|c|}{ Exportaciones } & \multicolumn{2}{|c|}{ Importaciones } & \multirow{2}{*}{ Fuente } \\
\hline Variable & Signo esperado & Variable & Signo esperado & \\
\hline$Q \exp$ & & Qimp & & $\begin{array}{l}\text { Banco de la } \\
\text { República }\end{array}$ \\
\hline$I T C R_{t}, I T C R_{t-1}$ & + & $I T C R_{t}, I T C R_{t-1}$ & - & $\begin{array}{c}\text { FMI, DANE, } \\
\text { DAS, Banco } \\
\text { de la } \\
\text { República } \\
\end{array}$ \\
\hline Yext $_{t}$, Yext $_{t-1}$ & + & $\mathrm{Ycol}_{t}, \mathrm{Ycol}_{t-1}$ & + & FMI \\
\hline$T_{1}, T_{4}$ & + & $T_{1}, T_{4}$ & + & \\
\hline
\end{tabular}

Fuente: Diseño de los autores.

\section{VERIFICACIÓN DE ESTACIONARIEDAD}

La presencia de no estacionariedad en las series de datos impide que se realicen adecuados análisis generalizables a todo el período de tiempo que se esté considerando. En este apartado se verifica la existencia o no de esta situación y se corrige según el grado de integración que se detecte.

La herramienta econométrica que se utiliza es el test de raíces unitarias desarrollado por Phillips y Perron (1988), con tres rezagos de truncamiento para cada una de las tres posibles especificaciones que permiten formular tres hipótesis nulas respecto al posible proceso estocástico que sigue cada una de las series tiempo.

En el cuadro 2 se presentan los resultados arrojados por el test de raíces unitarias. Las celdas grises señalan los valores del estadístico de PhillipsPerron que son menores a los valores críticos de MacKinnon, esto indica la existencia de no estacionariedad y hace suponer que todas las series, excepto las variables dicótomas de los trimestres, siguen un proceso estocástico integrado de primer orden o $X_{i t} \sim I(1)$ (la i-ésima variable $X$ en el t-ésimo momento del tiempo), el problema expuesto se corrige atendiendo a la literatura econométrica planteada en Guajarati $(2003,794)$ 
CUADRO No. 2. PRUEBA DE RAÍCES UNITARIAS

\begin{tabular}{|c|c|c|c|c|}
\hline \multirow[b]{2}{*}{ Variable } & \multirow[b]{2}{*}{ Especificación } & \multicolumn{3}{|c|}{ Estadístico de Phillips-Perron } \\
\hline & & Caminata aleatoria * & $\begin{array}{c}\text { Caminata aleatoria con } \\
\text { variaciones } \dagger\end{array}$ & $\begin{array}{c}\text { Caminata aleatoria con } \\
\text { variaciones alrededor } \\
\text { de una tendencia } \\
\text { estocástica } \ddagger\end{array}$ \\
\hline \multirow{3}{*}{$\ln \_$itcr } & Nivel & $p>0$ & $-2,44$ & $-1,94$ \\
\hline & Primeras diferencias & $-4,88$ & $-4,76$ & $-5,57$ \\
\hline & Segundas diferencias & $-10,39$ & $-10,51$ & $-10,26$ \\
\hline \multirow{3}{*}{$\ln \_q x$} & Nivel & $\mathrm{p}>0$ & $-0,54$ & $-1,77$ \\
\hline & \begin{tabular}{|l|} 
Primeras diferencias \\
\end{tabular} & $-7,04$ & $-7,55$ & $-7,74$ \\
\hline & Segundas diferencias & $-21,23$ & $-20,80$ & $-20,31$ \\
\hline \multirow{3}{*}{ ln_qimp } & Nivel & $\mathrm{p}>0$ & $-0,59$ & $-2,24$ \\
\hline & Primeras diferencias & $-6,06$ & $-6,72$ & $-6,73$ \\
\hline & Segundas diferencias & $-14,90$ & $-14,51$ & $-14,10$ \\
\hline \multirow{3}{*}{$\ln \_y x$} & Nivel & $p>0$ & $p>0$ & $-1,47$ \\
\hline & Primeras diferencias & $-3,88$ & $-4,47$ & $-4,66$ \\
\hline & Segundas diferencias & $-9,77$ & $-9,58$ & $-9,35$ \\
\hline \multirow{3}{*}{ ln_yn } & Nivel & $p>0$ & $-1,27$ & $-1,40$ \\
\hline & \begin{tabular}{|l|} 
Primeras diferencias \\
\end{tabular} & $-4,65$ & $-4,58$ & $-5,75$ \\
\hline & Segundas diferencias & $-10,72$ & $-10,90$ & $-10,63$ \\
\hline \multirow{3}{*}{ t1 } & Nivel & $\mathrm{p}>0$ & $-7,62$ & $-7,49$ \\
\hline & Primeras diferencias & $-41,13$ & $-40,24$ & $-39,00$ \\
\hline & Segundas diferencias & $-43,50$ & $-42,67$ & $-40,65$ \\
\hline \multirow{3}{*}{ t3 } & Nivel & $-5,66$ & $-21,03$ & $-21,36$ \\
\hline & \begin{tabular}{|l|} 
Primeras diferencias \\
\end{tabular} & $-32,08$ & $-31,40$ & $-30,96$ \\
\hline & Segundas diferencias & $-40,23$ & $-39,74$ & $-38,12$ \\
\hline \multirow{3}{*}{$\mathrm{t} 4$} & Nivel & $-5,66$ & $-25,37$ & $-24,83$ \\
\hline & Primeras diferencias & $-32,08$ & $-31,40$ & $-30,99$ \\
\hline & Segundas diferencias & $-47,46$ & $-46,94$ & $-45,25$ \\
\hline \multicolumn{5}{|c|}{$\begin{array}{l}\text { Nota: Valores críticos de MacKinnon al } 1 \%, 5 \% \text { y } 10 \% \text { de nivel de significancia, } \\
\text { respectivamente son: } \\
*: 2,64,-1,95 \text { y }-1,62 \\
\text { t: }-3,65,-2,96 \text { y }-2,61 \\
\ddagger:-4,27,-3,56 \text { y }-3,21\end{array}$} \\
\hline
\end{tabular}

Fuente: Diseño de los autores

\section{RESULTADOS Y COMENTARIOS}

Corregido el problema de raíces unitarias y efectuada la estimación econométrica, la evidencia permite afirmar que la demanda de exportaciones de turismo corresponde a la ecuación 311 :

$$
\begin{aligned}
& \underset{\mathrm{P} \text { value }(\mathrm{t})}{\Delta \ln Q \exp _{t}}=\underset{(0,0154)}{0,0499-0} \underset{(0,3226)}{0,0832 \Delta \ln I T C R_{t}}-\underset{(0,4658)}{0,0590 \Delta \ln I T C R_{t-1}}+\underset{(0,0323)}{2,1326 \Delta \ln Y e x t_{t}-0,7989 \Delta \ln Y e x t_{t-1}} \\
& \underset{(0,0000)}{-0,1621 T_{1}}+\underset{(0,5739)}{0,0154 T_{3}}-\underset{(0,6925)}{*} \\
& n=32 \quad R^{2}=0,7782 \quad F=12,0290 \quad \text { P value }(\mathrm{F})=0,0000 \\
& \sum \mathrm{e}^{2}=0,0611 \quad \text { Schwarz }=-2,5566 \quad \text { Akaike }=-2,9231
\end{aligned}
$$

Y la demanda estimada de importaciones colombianas de turismo a la ecuación 4:

11 Los asteriscos $\left(^{*}\right)$ encima de las variables indican que no corresponde su signo al esperado. 


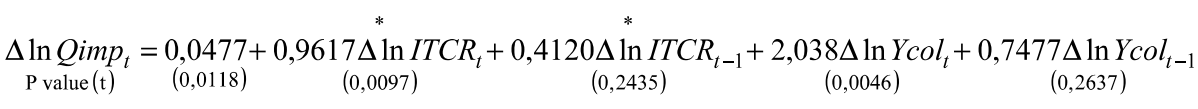

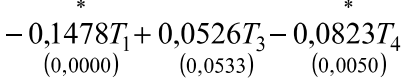

$$
\begin{aligned}
& n=32 \quad R^{2}=0,8011 \quad F=13,8137 \quad \text { P value }(\mathrm{F})=0,0000 \\
& \sum \mathrm{e}^{2}=0,0570 \quad \text { Schwarz }=-2,6260 \quad \text { Akaike }=-2,9924
\end{aligned}
$$

La estimación se llevó a cabo estimando 4 modelos adicionales, tanto para las exportaciones como para las importaciones de turismo, que incluían rezagos de orden superior. Estos modelos no eran mejores que los que se presentan más arriba (con base en los criterios de información de Akaike y Schwarz), por tanto no se toman en cuenta en el análisis.

Los coeficientes estimados representan elasticidades promedio. Aquí se confirma que el "producto" turismo es un bien de lujo, tanto para los extranjeros que llegan a Colombia como para los colombianos que hacen turismo en el exterior, dado que para ambos casos la elasticidad ingreso es mayor que la unidad. También nótese que los exportadores e importadores de turismo no tienen muy en cuenta su ingreso en un trimestre anterior a la hora de tomar sus decisiones, dado que para ambos casos no hay significancia estadística en los coeficientes.

Una hipótesis que se asumía era que la positiva situación de la economía mundial permitía un incremento en el gasto que los individuos efectuaban en turismo. Empíricamente se ha confirmado la existencia de un efecto ingreso para los demandantes extranjeros de turismo, pues aunque los precios relativos son mayores, debido principalmente al fenómeno de la revaluación del tipo de cambio peso-dólar, los demandantes continúan exportando turismo colombiano, de aquí que quizás este elemento no sea tan decisivo a la hora de decidirse por elegir a Colombia como destino turístico.

Los turistas colombianos que deciden viajar al extranjero están aprovechando la coyuntura revaluativa. Aunque cabría de esperar que ante un contexto de baja inflación interna los nacionales demandaran menos turismo extranjero, observable a través de una relación inversa entre ITCR y demanda de importaciones, la evidencia muestra lo contrario.

\section{CONCLUSIONES}

La actividad turística es de fundamental importancia en la economía de una nación por los diferentes impactos que tiene en otros sectores económicos. 
En el mundo se está viviendo cierto auge en la actividad, siendo Colombia no ajena a esto.

El fenómeno de la revaluación del tipo de cambio se constituye en un hecho muy importante para entender la dinámica del turismo. Con el presente trabajo se hizo una contribución a lo anterior en tanto que empíricamente se evaluó cómo la revaluación está afectando la actividad turística. Básicamente a lo que se llegó, luego de la estimación de un modelo a través del método $\mathrm{MCO}$, fue que para los turistas extranjeros los precios relativos no resulta ser determinantes a la hora de decidir considerar a Colombia como destino turístico. Sus decisiones dependen, elásticamente, del nivel de ingresos.

Para el caso de los colombianos que se deciden por turismo del exterior, se observa que están aprovechando, ceteris paribus, el contexto revaluativo, incluso cuando el nivel de precios nacionales está disminuyendo.

El presente trabajo deja mucho camino por recorrer en la profundización de la temática revaluación-turismo. También constituye un aliciente para que se construyan de bases de datos y fuentes de información, afines al turismo, de mayor calidad y de amplia cobertura; los resultados y las conclusiones que pudieran extraerse de cualquier estudio hallan limitantes en lo anterior. 


\section{REFERENCIAS BIBLIOGRÁFICAS}

Aguilera, M., Galvis, L. A. (1999). Determinantes de la demanda por turismo hacia Cartagena 1987-1998. Documentos de Trabajo Sobre Economía Regional, $N^{\circ}$ 9, marzo.

Banco De La República. Informe de la Junta Directiva al Congreso de la República. (2007) Julio [fecha de acceso 22 de agosto de 2007]. URL disponible en: http://www.banrep.gov.co/documentos/juntadirectiva/ informe-congreso/2007/infor me_al_c ongreso_jul_07.pdf.

CEPAL (2007). Anuario estadístico de América Latina y el Caribe, 2006, notas técnicas [en línea] [fecha de acceso 10 de septiembre de 2007]; URL disponible en: http://www.eclac.org/publicaciones/xml/3/28063/LCG2332B_nota s_tecnicas.pdf.

DANE (2007). Análisis de los resultados de la Encuesta de Turismo a Hogares 2003. [Documento en formato .pdf]. Bogotá D. C.: DANE; 2007.

Espasa, A., Gómez Churruca R., Morales E. (1993). Un análisis econométrico del turismo de España: implicaciones para el estudio sectorial de las exportaciones y algunas consideraciones de política económica. Espasa, A. y Canceló, J. R. (eds), Métodos cuantitativos para el análisis de la coyuntura económica, Madrid, Alianza, 605-656.

Greene, W. (2003). Econometric analisys. 5 ed, New Jersey, Prentice Hall.

Gujarati, D. (2003). Econometría. 4 ed, México D.F., McGraw Hill.

Halicioglu, F. (2004). An ARDL model of aggregate tourism demand for Turkey. Global Business and Economics Review (Antology), 614-624. URL disponible en: http://129.3.20.41/eps/it/papers/0503/0503005.pdf.

Mantero, R., Perelmuter N., Sueiro I. (2004). Determinantes económicos del turismo receptivo en Uruguay: ¿aporta información relevante un análisis desagregado? URL disponible en: http://www.bcu.gub.uy/autoriza/peiees/jor /2004/iees03j3430804.pdf. 
OMT (2006). Datos esenciales del turismo. [En línea] 2006 [fecha de acceso 30 de agosto de 2007]. URL disponible en:http://www.unwto.org/facts/eng/pdf/highlights/highli ghts06_sp_lr.pdf.

Phillips, P., Perron, P. (1988). Testing for a unit root in time series regression. Biometrika, 75, 2, 335-346.

Rodríguez Feijoó, S., Dávila Quintana D., Rodríguez Caro, A., Tejera Gil, M. (2003). Determinantes de la demanda de turismo vacacional en las Islas Canarias. Investigaciones Regionales, 3, 81-98. 


\section{ANEXO 1:}

\section{A) CONSTRUCCIÓN DE UN ÍNDICE DE COMPETITIVIDAD}

El presente anexo describe el proceso mediante el cual se construyó el índice de tipo de cambio real para Colombia empleado en este documento, que se tomará como indicador de competitividad.

La metodología empleada es muy similar a la del Banco de la República ${ }^{12}$, la diferencia radica en la forma en la que se calcula el ponderador $W_{i j, t}$. El Banco emplea ponderaciones móviles mensuales de orden 12 del comercio total entre Colombia y sus principales socios comerciales. Para el presente trabajo se utilizaron ponderaciones móviles trimestrales de orden 4 entre Colombia y los países considerados como los principales 5 competidores en materia turística OMT $(2006,8)^{13}$.

El ITCR viene dado del cálculo de:

$$
\operatorname{ITCR}_{i, t}=\frac{\left(\prod_{j=1}^{n}\left(I E_{i j, t}\right)^{W_{j, t}}\right)\left(\prod_{j=1}^{n}\left(I P_{j, t}\right)_{W_{j, t}}\right)}{\left(I P_{i, t}\right)}
$$

De donde $I E_{i j, t}$ es un índice de tasa de cambio entre Colombia $(i)$ y el jésimo país competidor en el trimestre $t . I P_{j, t}$ es el índice de precios al consumidor en cada uno de los $j$ países en cada trimestre. $I P_{i . t}$ hace referencia al índice de precios al consumidor de Colombia. Todos los índices expresados en base 2000. $W_{i j, t}$ corresponde al ponderador dado por:

$$
W_{i, t}=\frac{\sum_{k=0}^{3}(X+M)_{j, t-k}}{\sum_{j=1}^{n} \sum_{k=0}^{3}(X+M)_{j, t-k}}
$$

Con $X$ y $M$ siendo respectivamente las cantidades exportadas de turismo en Colombia por parte de personas extranjeras y las cantidades importadas de turismo por parte de personas nacionales, teniendo como referencia los principales países competencia turística de Colombia.

${ }^{12}$ Que aparece en Huertas et al (2003).

${ }^{13}$ Esto aparece en el apartado sobre Índice de precios relativos en el cuerpo del artículo. 


\section{B) CONSTRUCCIÓN DE UN ÍNDICE DE RENTA REAL}

Con base en las recomendaciones y la metodología de Espasa, Gómez y Morales $(1993,637-638,650-651,654)$ se construyó un índice de renta real agragada, que incluyera los diferentes países que mantienen relaciones comerciales de tipo turístico con Colombia vía demanda, este se usó como variable explicativa en el modelo de exportaciones estimado en este trabajo; también se elaboró otro sólo para Colombia, que fue añadido en el modelo de importaciones turísticas.

El proceso de construcción comenzó con calcular la expresión:

$$
Y n=\prod_{i=1}^{N} Y_{i t}^{\alpha_{i t}}
$$

En la que $Y n$ corresponde al índice de renta real agregada, $Y_{i t}$ es un indicador del PIB a precios del base 2000 del i-ésimo país, del total $N$, en el año $t$ que demanda turismo colombiano. $\alpha_{i t}$ es un ponderador que se calcula a través de:

$$
\begin{aligned}
& \alpha_{i t}=\frac{n_{i t}}{\sum_{i=1}^{N} n_{i t}} \\
& n_{i t}=\frac{T_{i t} y_{i 2000}}{\sum_{i=1}^{N} y_{i 2000}}
\end{aligned}
$$

En las expresiones anteriores $T_{i t}$ es el número de turistas acumulados en los tres trimestres anteriores, incluyendo el trimestre actual. El factor $y_{i 2000}$ corresponde al PIB per cápita real $(2000=100)$ del i-ésimo país, esto se hace con el propósito de normalizar los diferentes valores de cantidades de turistas de los diferentes países y permitir comparaciones. Finalmente $n_{i t}$ es el número de turistas normalizados procedentes del país $i$ en el t-ésimo trimestre. 


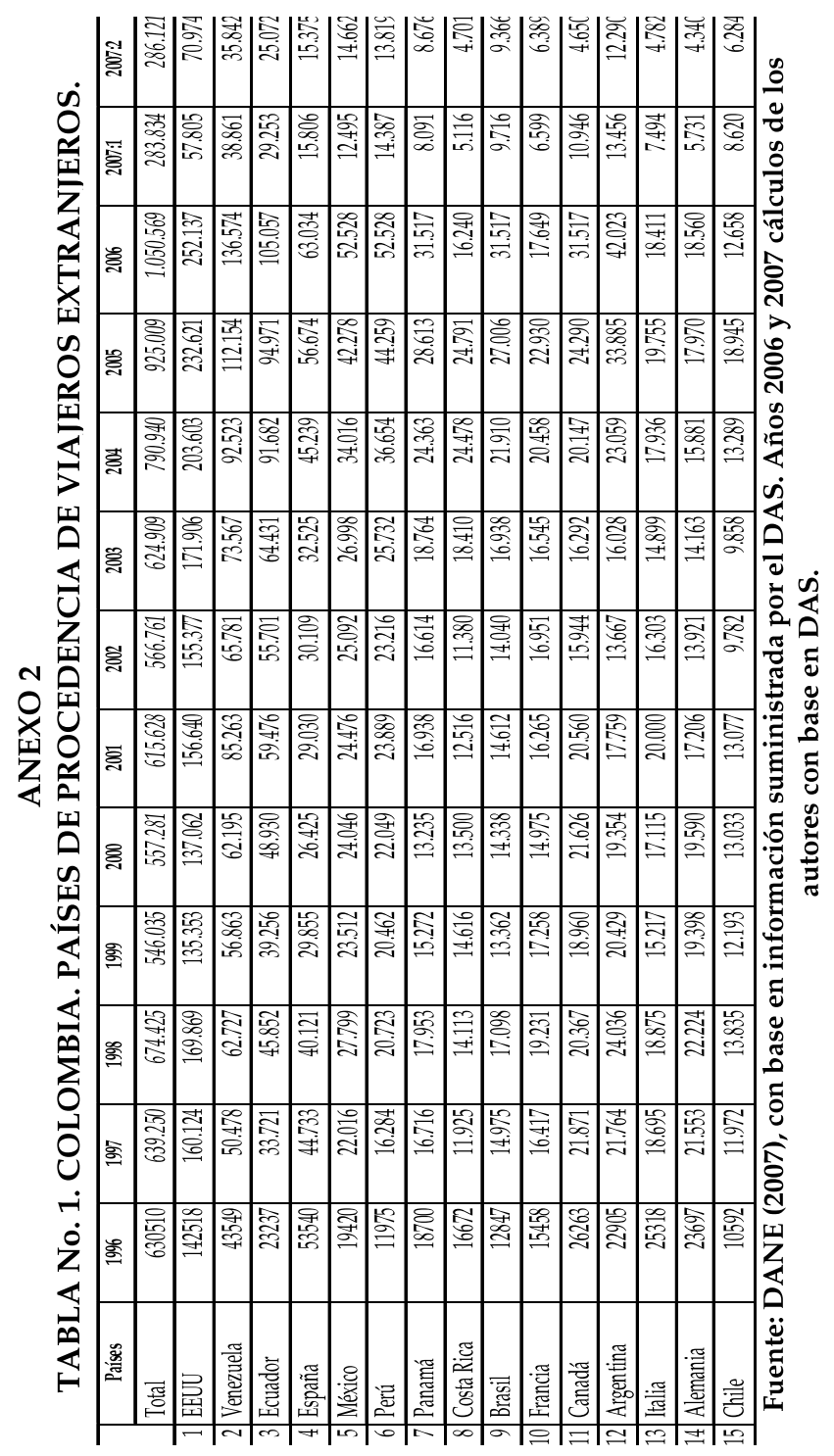




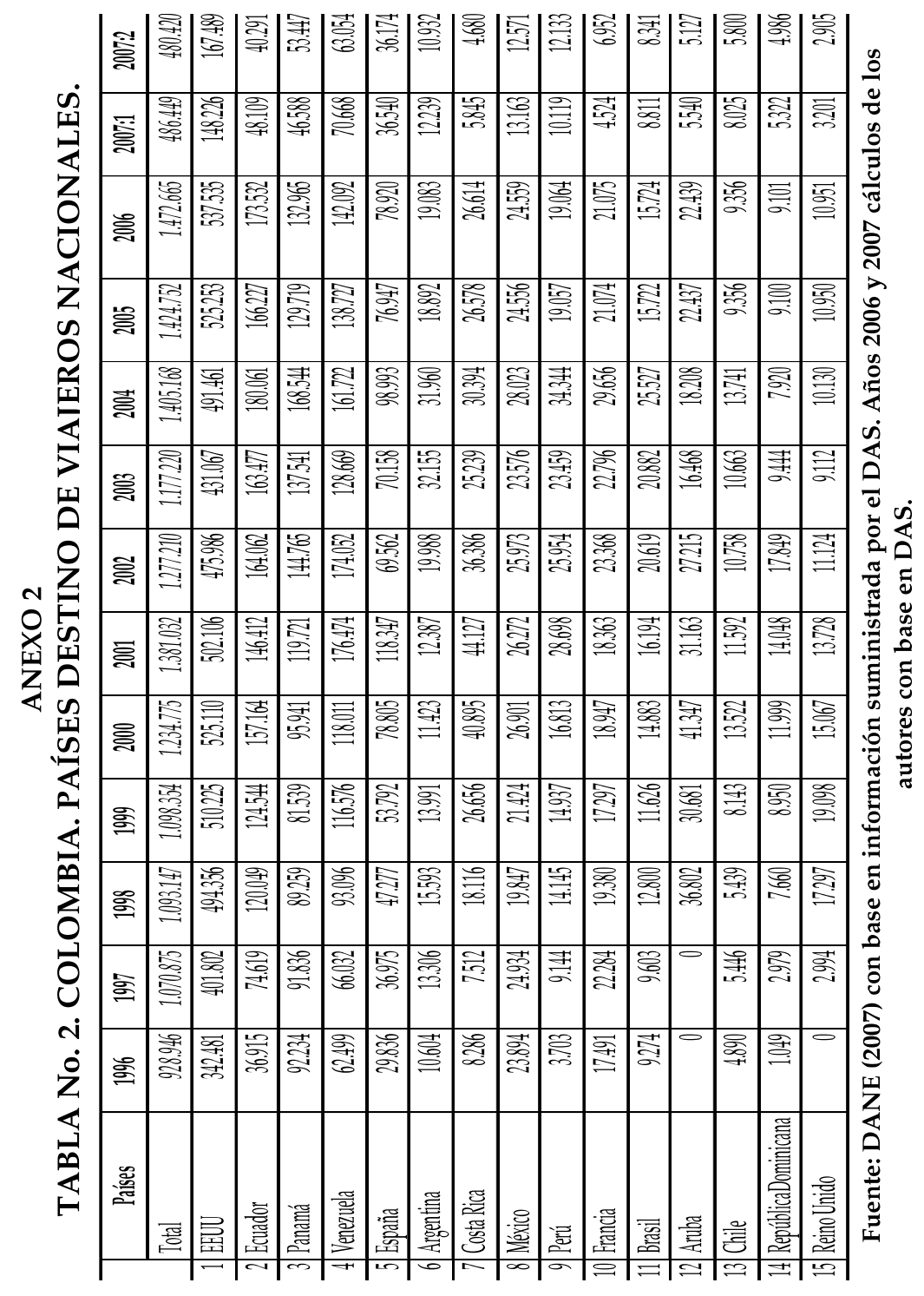

